\title{
ESTUDO DA INFLUÊNCIA DO DILUENTE REATIVO PGE NA CINÉTICA DE CURA DE RESINA EPÓXI UTILIZADA EM COMPÓSITOS ESTRUTURAIS
}

\author{
Michelle Leali Costa \\ Departamento de Física - Instituto Tecnológico de Aeronáutica - Centro Técnico Aeroespacial - São José dos Campos - SP \\ Mirabel Cerqueira Rezende e Luiz Claudio Pardini \\ Divisão de Materiais - Instituto de Aeronáutica e Espaço - Centro Técnico Aeroespacial - São José dos Campos - SP
}

\begin{abstract}
EFFECT ON THE REACTIVE DILUENT PGE ON THE CURE KINETICS OF AN EPOXY RESIN USED IN STRUCTURAL COMPOSITES. Phenil glycidyl ether (PGE), a monofunctional diluent, has been used in epoxy resins formulations in order to increase the toughness of the epoxy molded composite. In a systematic study concerning its influence in the cure kinetics of the epoxy resin, it was used in concentrations of 2,$5 ; 5,0 ; 10$ and $20 \%$ in relation to a diglycidyl ether bisphenol-A (DGEBA)/diamino diphenil-sulfone (DDS) base matrix. Dynamic and isothermal scanning analysis were carried out using a differential scanning calorimety (DSC) equipment. For all the concentrations of PGE, a n order kinetics was observed, with $n$ varing between $0,35-0,91$ as a function of the increase in the PGE concentration.
\end{abstract}

Keywords: kinetic of cure; epoxy resin; reactive diluent.

\section{INTRODUÇÃO}

Atualmente, a diversidade de materiais disponíveis para uso em engenharia é extraordinariamente grande, atendendo às mais diversas aplicações do mercado. Dentro desse contexto, os compósitos apresentam-se como um caso de reconhecido interesse em materiais de engenharia não convencionais ${ }^{1}$.

Os compósitos poliméricos estruturais têm como características a alta resistência (>800 MPa), o alto módulo de elasticidade $(>50 \mathrm{GPa})$ e a baixa massa específica $\left(\sim 2 \mathrm{~g} / \mathrm{cm}^{3}\right)^{2,3}$. Esses materiais são obtidos utilizando-se como matéria-prima básica préimpregnados, que são constituídos basicamente de fibras de reforço aglutinadas em orientações pré-determinadas por uma matriz polimérica. Os pré-impregnados são, por sua vez, moldados em autoclave na conformação de elementos estruturais ${ }^{4-6}$.

É, portanto, de fundamental importância um controle adequado dos parâmetros de processamento do pré-impregnado, de modo que a peça obtida atenda aos requisitos de qualidade aeronáutica.

A tecnologia de fabricação de pré-impregnados é controlada por poucas empresas fornecedoras da indústria aeronáutica, que manipulam as formulações do sistema de resina de modo que estas atendem necessidades específicas em termos de propriedades mecânicas do compósito final. O objetivo do presente trabalho, levando-se em consideração o exposto, é estudar a cinética de cura de uma resina epóxi formulada com diferentes porcentagens de diluente reativo fenilglicidiléter (PGE), via calorimetria diferencial de varredura (DSC), cujos parâmetros vão auxiliar tanto no processamento de pré-impregnados, quanto na moldagem final de compósitos de uso aeronáutico.

$\mathrm{Na}$ área de compósitos estruturais, a técnica de DSC é uma das mais utilizadas para a determinação de temperaturas de transição vítrea, ponto de fusão, etc., bem como no estudo da cinética de polimerização da matriz impregnante das fibras de reforço. Este ponto é fundamental no processamento de pré-impregnados, pois é a cinética de cura que vai delinear o ciclo de cura a ser utilizado, ou seja, qual o perfil de temperatura que deve ser aplicado na fabricação do compósito. Existem dois métodos de análise térmica por DSC que permitem o estudo da cinética de cura: os métodos dinâmicos e os métodos isotérmicos ${ }^{7,8}$.

Utilizando-se o método isotérmico é possível identificar dois tipos de reação: o de ordem $\mathbf{n}$ e o de ordem autocatalítica ${ }^{9}$. Se o pico máximo da isoterma estiver próximo a $\boldsymbol{t}=\boldsymbol{0}$, o sistema obedece a uma cinética de ordem $\boldsymbol{n}$ e pode ser estudado pelo método dinâmico ou pelo método isotérmico. Caso o pico máximo se forme entre 30 e $40 \%$ do tempo total da análise, o sistema é autocatalítico e deve ser estudado exclusivamente pelo método isotérmico ${ }^{7-12}$

$\mathrm{O}$ modelo de ordem $\mathrm{n}$ assume que a reação obedece a equação 1:

$\mathrm{v}=\frac{d \alpha}{d t}=k(1-\alpha)^{n}$

onde v é a velocidade da reação, k é a constante de velocidade e $\boldsymbol{\alpha}$ é o grau de conversão em um dado tempo $\boldsymbol{t}$. Aplicando-se logarítmos e graficando $\ln (\mathrm{d} \alpha / \mathrm{dt})$ versus $\ln (1-\alpha)$, obtem-se uma reta cuja inclinação representa o valor de $\mathbf{n}$ e a interseção com o eixo x o valor de $\mathbf{l n} \mathbf{k}$.

Os dois parâmetros $\boldsymbol{d} \boldsymbol{\alpha} / \boldsymbol{d} \boldsymbol{t} \boldsymbol{e} \boldsymbol{\alpha}$ necessários para os cálculos são obtidos das isotermas de DSC. A taxa de reação é obtida pela divisão do termo $\boldsymbol{d H} / \boldsymbol{d} \boldsymbol{T}$ (calor de reação), a uma temperatura $\boldsymbol{T}$, pela entalpia total $\boldsymbol{\Delta H}_{\boldsymbol{0}}$ (equação 2):

$\frac{d \alpha}{d t}=(d H / d t) / \Delta \mathrm{H}_{0}$

onde $\Delta \boldsymbol{H}_{0}$ é a entalpia experimental correspondente à área total do pico ou à entalpia teórica encontrada na literatura.

$\mathrm{O}$ valor de $\boldsymbol{\alpha}$ é determinado pela medida do calor de reação parcial no tempo $\boldsymbol{t}$, dividindo-se $\boldsymbol{\Delta} \boldsymbol{H}_{\boldsymbol{p}}$ pelo calor total da reação isotérmica, $\boldsymbol{\Delta} \boldsymbol{H}_{\text {iso }}$, fornecendo a equação 3.

$\alpha=\frac{\Delta \mathrm{H}_{\mathrm{p}}}{\Delta \mathrm{H}_{i s o}}$

Finalmente, com um mínimo de três isotermas é possível construir um gráfico de Arrhenius, de onde obtêm-se os parâmetros cinéticos $\boldsymbol{E}$ (energia de ativação) da inclinação da reta, $\boldsymbol{e} \boldsymbol{A}$ (fator pré-exponencial) da interseção com o eixo x. 


\section{PARTE EXPERIMENTAL}

\section{Materiais}

\section{Resina base}

A resina epóxi utilizada é a baseada em éter diglicidílico do bisfenol-A (DGEBA), cujo nome comercial é D.E.R. 331, da Dow Produtos Químicos Ltda ${ }^{13}$, mostrada na Figura 1.<smiles>CC(C)(OCC1CO1)C(O)COC(C)(C)C(C)(C)OCC1CO1</smiles>

Figura 1. Fórmula estrutural da resina DGEBA.

\section{Agente de cura}

O agente de cura utilizado é o difenil diamino sulfona (DDS), cujo nome comercial é HT 976, da Ciba Geigy Química S/A. O DDS é um agente de cura em pó, de cor rósea, do tipo amina aromática, que deve ser fundido para sua utilização. A Tabela 1 lista as principais características físicas deste agente de cura. A Figura 2 mostra a sua fórmula química estrutural.

Tabela 1. Propriedades do DDS ${ }^{13}$.

\begin{tabular}{cc}
\hline Ponto de fusão $(\cdot \mathrm{C})$ & 175 \\
Massa de hidrogênio ativo $(\mathrm{g} / \mathrm{mol})$ & 57 \\
Massa molar $(\mathrm{g} / \mathrm{mol})$ & 248 \\
\hline
\end{tabular}

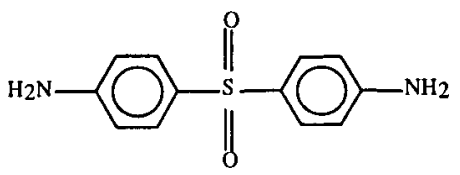

Figura 2. Fórmula estrutural do DDS.

\section{Diluente reativo}

O diluente reativo utilizado é o PGE, trata-se de uma resina epóxi alifática de poliglicol. Este diluente quando combinado com resinas convencionais confere flexibilidade, alongamento e melhor resistência ao impacto ao polímero curado. O seu nome comercial é D.E.R.732, da Dow Produtos Químicos Ltda e a sua fórmula química estrutural está representada na Figura 3.

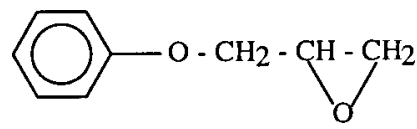

Figura 3. Fórmula química estrutural do PGE.

\section{Preparação das formulações}

As formulações estudadas foram preparadas em um becker. À resina DGEBA foi adicionado o agente de cura, endurecedor, em quantidade estequiométrica, em relação à massa correspondente de resina. Em seguida, foram preparadas as formulações de resina/agente de cura aditadas com as proporções de diluente a 2,5; 5,0; 10 e $20 \%$ em relação à massa total da formulação. As formulações foram, então, aquecidas à temperatura de fusão do endurecedor sob agitação manual, por aproximadamente 10 minutos, sendo em seguida resfriadas em um banho de gelo por igual intervalo de tempo. Assim, foram obtidas cinco formulações:

- DGEBA(100)/DDS(30) (peso/peso (p/p));

- DGEBA/DDS/PGE 2,5\%, denominada de maneira simplificada de PGE 2,5\%;

- DGEBA/DDS/PGE 5,0\% ou PGE 5\%;

- DGEBA/DDS/PGE $10 \%$ ou PGE $10 \%$;

- DGEBA/DDS/PGE $20 \%$ ou PGE $20 \%$.

\section{Caracterização}

Para os ensaios foi utilizado o analisador térmico PerkinElmer-7 Series Thermal Analysis System, previamente calibrado com índio, sob fluxo constante de nitrogênio $(100 \mathrm{~mL} / \mathrm{min})$ e taxa de aquecimento de $10^{\circ} \mathrm{C} / \mathrm{min}$.

$\mathrm{O}$ peso das amostras variou de $10 \mathrm{a} 15 \mathrm{mg}$ de modo a não provocar o vazamento das mesmas durante a análise permitindo, assim, a obtenção de dados reprodutíveis. Foram utilizados porta-amostras de alumínio do tipo selado.

Para cada formulação foram realizadas varreduras dinâmicas (Tabela 2), a partir das quais foi obtido o calor total da reação $\Delta \mathrm{H}_{0}$ e estabelecidas as temperaturas isotérmicas para o estudo da cinética de cada sistema. A varredura isotérmica forneceu o valor do calor parcial da reação, $\Delta \mathrm{H}_{\mathrm{p}}$.

$\mathrm{O}$ programa computacional Isothermal Kinetics existente no módulo central do equipamento Perkin Elmer, permite a determinação de variáveis relacionadas com a cinética de cura de cada formulação. Este programa computacional também possibilita obter a ordem de reação que melhor se adapta ao sistema em estudo ${ }^{14}$.

\section{RESULTADOS E DISCUSSÃO}

A Tabela 3 apresenta os resultados das varreduras dinâmicas das formulações DGEBA/DDS; PGE2,5\%, PGE5\%, PGE10\% e PGE 20\%. Observa-se que, a temperatura inicial de polimerização das formulações DGEBA/DDS, PGE2,5\% e PGE5\% é aproximadamente a mesma $\left(123 \pm 1^{\circ} \mathrm{C}\right)$.

As temperaturas médias de pico das cinco formulações são aproximadamente da ordem de $226 \pm 1{ }^{\circ} \mathrm{C}$ (Tabela 3). Este comportamento é esperado, uma vez que o agente de cura utilizado nos cinco sistemas foi o mesmo (DDS) sugerindo, assim, que as temperaturas de polimerização devem ser próximas. O que varia é a temperatura final de cura e o desprendimento de calor envolvido em cada formulação, como pode ser observado nos

Tabela 2. Condições térmicas utilizadas na obtenção das curvas de DSC.

\begin{tabular}{|c|c|c|c|c|c|}
\hline $\begin{array}{c}\text { Curvas de DSC } \\
\text { realizadas }\end{array}$ & $\begin{array}{l}\text { DGEBA } \\
\text { DDS }\end{array}$ & $\begin{array}{c}\text { DGEBA } \\
\text { DDS/ PGE } \\
2,5 \%\end{array}$ & $\begin{array}{c}\text { DGEBA } \\
\text { DDS/PGE } \\
5 \%\end{array}$ & $\begin{array}{c}\text { DGEBA } \\
\text { DDS/PGE } \\
10 \%\end{array}$ & $\begin{array}{c}\text { DGEBA } \\
\text { DDS/PGE } \\
20 \%\end{array}$ \\
\hline Varredura dinâmica & $75-330^{\circ} \mathrm{C}$ & $75-330^{\circ} \mathrm{C}$ & $75-330^{\circ} \mathrm{C}$ & $75-330^{\circ} \mathrm{C}$ & $75-330^{\circ} \mathrm{C}$ \\
\hline Isoterma 1 & $160^{\circ} \mathrm{C}$ & $170^{\circ} \mathrm{C}$ & $170^{\circ} \mathrm{C}$ & $170^{\circ} \mathrm{C}$ & $170^{\circ} \mathrm{C}$ \\
\hline Isoterma 2 & $170^{\circ} \mathrm{C}$ & $180^{\circ} \mathrm{C}$ & $180^{\circ} \mathrm{C}$ & $180^{\circ} \mathrm{C}$ & $180^{\circ} \mathrm{C}$ \\
\hline Isoterma 3 & $190^{\circ} \mathrm{C}$ & $190^{\circ} \mathrm{C}$ & $190^{\circ} \mathrm{C}$ & $190^{\circ} \mathrm{C}$ & $190^{\circ} \mathrm{C}$ \\
\hline Isoterma 4 & $200^{\circ} \mathrm{C}$ & $200^{\circ} \mathrm{C}$ & $200^{\circ} \mathrm{C}$ & $200^{\circ} \mathrm{C}$ & $200^{\circ} \mathrm{C}$ \\
\hline
\end{tabular}


Tabela 3. Resultados das varreduras dinâmicas dos sistemas em estudo e a relação entre os $\Delta \mathrm{H}_{0}$ das varreduras para as cinco formulações com DDS.

\begin{tabular}{cccccc}
\hline Formulações & $\mathrm{T}_{\text {inicial }}\left({ }^{\circ} \mathrm{C}\right)$ & $\mathrm{T}_{\text {pico }}\left({ }^{\circ} \mathrm{C}\right)$ & $\mathrm{T}_{\text {final }}\left({ }^{\circ} \mathrm{C}\right)$ & $\Delta \mathrm{H}_{0}(\mathrm{~J} / \mathrm{g})$ & $\Delta \mathrm{H}_{0} \mathrm{n} / \Delta \mathrm{H}_{0}(\mathrm{~J} / \mathrm{g})$ \\
\hline DGEBA/DDS & $123 \pm 1,0$ & $225 \pm 1,0$ & $298 \pm 1,0$ & -271 & 1,00 \\
DGEBA/DDS/PGE 2,5\% & $123 \pm 1,0$ & $225 \pm 1,0$ & $310 \pm 1,0$ & -368 & -315 \\
DGEBA/DDS/PGE 5,0\% & $123 \pm 1,0$ & $227 \pm 1,0$ & $321 \pm 1,0$ & -250 & 1,16 \\
DGEBA/DDS/PGE 10\% & $117 \pm 1,0$ & $228 \pm 1,0$ & $314 \pm 1,0$ & -230 & 0,92 \\
DGEBA/DDS/PGE 20\% & $116 \pm 1,0$ & $227 \pm 1,0$ & $312 \pm 1,0$ & -73 \\
\hline
\end{tabular}

$\mathrm{n}$ : formulações com 2,5, 5,0, 10 e $20 \%$ de PGE

valores de $\Delta \mathrm{H}$. A Tabela 3 mostra, também, a relação entre os $\Delta \mathrm{H}_{0}$ obtidos.

A relação entre os $\Delta \mathrm{H}_{0}$ dinâmicos, tomando-se como base a formulação somente com DDS, mostra que a adição de PGE nas concentrações de 2,5 e $5,0 \%$ aumenta a exotermia da reação, uma vez que uma maior quantidade de grupos epóxi é introduzida no sistema, aumentando, conseqüentemente, o valor de $\Delta \mathrm{H}_{0}$. Já no caso das formulações com 10 e $20 \%$ de PGE, o $\Delta \mathrm{H}_{0}$ diminui, provavelmente, devido à ocorrência de um maior número de interações ou reações paralelas.

A quantidade estequiométrica do agente de cura DDS, em relação à resina DGEBA, foi mantida fixa em todas as formulações, assim, com o aumento da concentração de PGE no sistema começou existir uma falta de grupos amínicos para que fosse realizada a cura. Neste ponto a fase final da polimerização passou a ocorrer também, por meio de outras reações incluindo interações entre grupos epóxi/epóxi. Assim, podem ocorrer reações entre DGEBA-DDS; DGEBA-PGE, DGEBADDS-PGE, DDS-PGE, etc., diminuindo, com isso, a exotermia da reação. Comportamento semelhante a esse já foi abordado na literatura ${ }^{10,13}$. Essas reações paralelas podem gerar uma fase discreta ou diferenciada na matriz alterando as características mecânicas da formulação.

Conforme apresentado, sabe-se que a ordem de reação de um sistema é definida de acordo com a região escolhida da curva de DSC em estudo ${ }^{10}$. O conjunto de isotermas obtido foi utilizado para caracterizar a ordem de reação das formulações DGEBA/DDS, PGE2,5\%, PGE5\%, PGE10\% e PGE20\%. A Figura 4 apresenta as curvas representativas da formulação DGEBA/DDS.

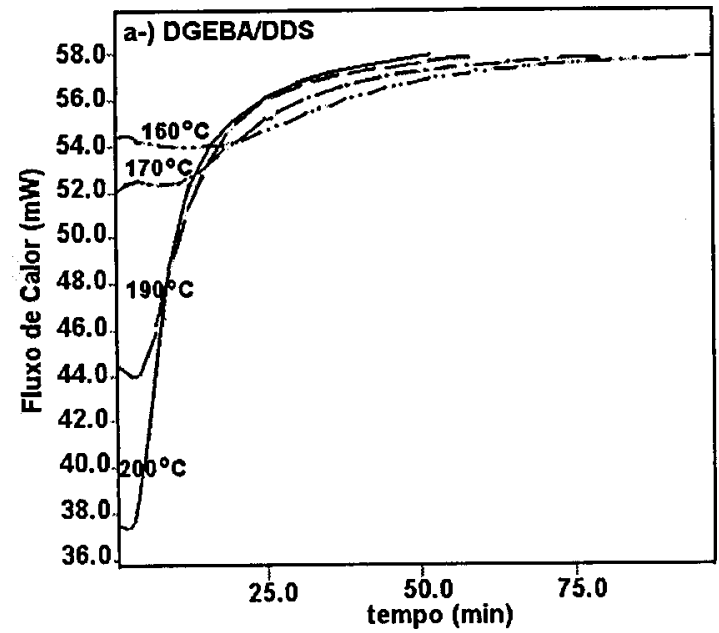

Figura 4. Isotermas a diferentes temperaturas obtidas por DSC representativas do sistema DGEBA/DDS.

Todas as curvas de DSC obtidas para as cinco formulações possuem o mesmo formato das curvas da Figura 4. Foi observado também que, a evolução de calor máxima, ou seja, a taxa de reação $(\mathrm{d} \alpha / \mathrm{dt})$ máxima ocorre logo no início da reação das formulações, próxima da conversão $\alpha \cong 0$. Portanto, todos os cinco sistemas obedecem a uma cinética de cura de ordem $n$

A cinética de ordem $n$ implica que o sistema obedece a equação 1 . A constante $n$, além de fornecer a ordem da reação e os dados necessários ao estudo do mecanismo de polimerização, descreve, também, o formato da isoterma (função exponencial), como pode ser observado na Figura 4. Logo, o valor de $n$ influencia na taxa de reação do sistema, que por sua vez está correlacionado aos parâmetros do ciclo de cura destinado ao processamento do compósito.

De posse dos dados da Tabela 3, da Figura 4 e das demais isotermas obtidas para as formulações contendo PGE foram calculados, por meio do programa Isothermal kinetics ${ }^{14}$, os resultados cinéticos apresentados nas Tabelas 4, 5, 6, 7 e 8 .

Tabela 4. Resultados de entalpia das isotermas $\left(\Delta \mathrm{H}_{\text {iso }}\right)$ e parâmetros cinéticos ( $\mathrm{k}$ e $\mathrm{n}$ ) do sistema DGEBA/DDS.

\begin{tabular}{cccc}
\hline $\mathrm{T}\left({ }^{\circ} \mathrm{C}\right)$ & $\Delta \mathrm{H}_{\text {iso }}(\mathrm{J} / \mathrm{g})$ & $\mathrm{k}(\mathrm{T})$ & $\mathrm{n}$ \\
\hline 160 & -194 & $4,50.10^{-4}$ & 0,38 \\
170 & -224 & $8,99.10^{-4}$ & 0,58 \\
190 & -260 & $1,43.10^{-3}$ & 0,78 \\
200 & -280 & $2,01.10^{-3}$ & 0,96 \\
\hline
\end{tabular}

Tabela 5. Resultados de entalpia das isotermas $\left(\Delta \mathrm{H}_{\text {iso }}\right)$ e parâmetros cinéticos ( $\mathrm{k}$ e $\mathrm{n}$ ) do sistema DGEBA/DDS/PGE2,5\%.

\begin{tabular}{cccc}
\hline $\mathrm{T}\left({ }^{\circ} \mathrm{C}\right)$ & $\Delta \mathrm{H}_{\text {iso }}(\mathrm{J} / \mathrm{g})$ & $\mathrm{k}(\mathrm{T})$ & $\mathrm{n}$ \\
\hline 170 & -173 & $2,60.10^{-4}$ & 0,38 \\
180 & -194 & $3,60.10^{-4}$ & 0,57 \\
190 & -222 & $5,19.10^{-3}$ & 0,72 \\
200 & -250 & $7,10.10^{-3}$ & 0,91 \\
\hline
\end{tabular}

Tabela 6. Resultados de entalpia das isotermas $\left(\Delta \mathrm{H}_{\text {iso }}\right)$ e parâmetros cinéticos (k e n) do sistema DGEBA/DDS/PGE5,0\%.

\begin{tabular}{cccc}
\hline $\mathrm{T}\left({ }^{\circ} \mathrm{C}\right)$ & $\Delta \mathrm{H}_{\text {iso }}(\mathrm{J} / \mathrm{g})$ & $\mathrm{k}(\mathrm{T})$ & $\mathrm{n}$ \\
\hline 170 & -160 & $7,03.10^{-4}$ & 0,36 \\
180 & -188 & $9,72.10^{-4}$ & 0,56 \\
190 & -210 & $1,38.10^{-3}$ & 0,61 \\
200 & -249 & $1,93.10^{-3}$ & 0,82 \\
\hline
\end{tabular}

Tabela 7. Resultados de entalpia das isotermas $\left(\Delta \mathrm{H}_{\text {iso }}\right)$ e parâmetros cinéticos (k e n) do sistema DGEBA/DDS/PGE10\%.

\begin{tabular}{cccc}
\hline $\mathrm{T}\left({ }^{\circ} \mathrm{C}\right)$ & $\Delta \mathrm{H}_{\text {iso }}(\mathrm{J} / \mathrm{g})$ & $\mathrm{k}(\mathrm{T})$ & $\mathrm{n}$ \\
\hline 170 & -147 & $6,36.10^{-4}$ & 0,36 \\
180 & -148 & $10,1.10^{-4}$ & 0,53 \\
190 & -164 & $1,47.10^{-3}$ & 0,57 \\
200 & -230 & $1,90.10^{-3}$ & 0,75 \\
\hline
\end{tabular}


Tabela 8. Resultados de entalpia das isotermas $\left(\Delta \mathrm{H}_{\text {iso }}\right)$ e parâmetros cinéticos (k e n) do sistema DGEBA/DDS/PGE20\%.

\begin{tabular}{cccc}
\hline $\mathrm{T}\left({ }^{\circ} \mathrm{C}\right)$ & $\Delta \mathrm{H}_{\text {iso }}(\mathrm{J} / \mathrm{g})$ & $\mathrm{k}(\mathrm{T})$ & $\mathrm{n}$ \\
\hline 170 & -109 & $6,45.10^{-4}$ & 0,35 \\
180 & -118 & $9,59.10^{-4}$ & 0,54 \\
190 & -147 & $1,44.10^{-3}$ & 0,55 \\
200 & -222 & $10,47.10^{-3}$ & 0,70 \\
\hline
\end{tabular}

Observa-se, a partir dos resultados das tabelas 4-8 que o valor de $\Delta \mathrm{H}$ isotérmico $\left(\Delta \mathrm{H}_{\mathrm{iso}}\right)$ variou dentro de um mesmo sistema, ou seja, o $\Delta \mathrm{H}$ aumentou em módulo com o aumento da temperatura.

Comparando-se os valores de $\mathrm{n}$ e $\mathrm{k}$ para um mesmo sistema, pode-se observar que, a ordem de reação e a constante de velocidade aumentaram com o aumento da temperatura. Isso ocorre pois o aumento da temperatura provoca uma distribuição diferente de velocidades (e de energias) seguido de um aumento no valor médio da energia das moléculas. Com isso, um número maior de moléculas adquire energia suficiente para, por colisão, vencer a barreira de ativação da reação e, conseqüentemente, a velocidade da reação aumenta ${ }^{10,13}$.

Verifica-se, também, que a ordem de reação dos sistemas em estudo diminuiu com o aumento da concentração de PGE, ou seja, as moléculas de PGE introduziram uma maior quantidade de grupos epóxi nas formulações, atuando como retardadoras da reação de polimerização. As moléculas de PGE passaram a concorrer com os grupos epóxi das moléculas de DGEBA, fazendo com que o DDS passasse a ser o reagente limitante da reação.

Essa maior proporção de reações paralelas, diminui os valores de $\mathrm{n}$, tornando a reação mais lenta. Entretanto, esse efeito consegue ser minimizado em temperaturas mais altas $\left(200^{\circ} \mathrm{C}\right)$, devido ao aumento do número total de colisões entre as moléculas reagentes aumentando, assim, a probabilidade de ocorrência da reação e conseqüentemente a velocidade da reação.

Com os resultados obtidos é possível conhecer os valores da taxa de reação e da conversão para as isotermas estudadas. A Figura 5 mostra a taxa de reação de cada isoterma obtida em função do tempo para os cinco sistemas. A análise desta Figura permite concluir que, para temperaturas superiores a $180^{\circ} \mathrm{C}$ as curvas exibem valores mais próximos para os máximos de taxa de reação. Comportamento semelhante foi observado nos gráficos da taxa de reação em função do grau de conversão.

Os valores próximos para os máximos de taxa de reação e de conversão são características que delimitam os mecanismos de reação dos tipos ordem n e autocatalítica ${ }^{15,16}$. Logo, os gráficos da Figura 5 confirmam a ordem de reação n para as formulações em estudo.

Nos gráficos da Figura 5 verifica-se que a temperatura tem grande influência sobre a taxa de reação. Em temperaturas mais baixas $\left(\sim 160-180^{\circ} \mathrm{C}\right)$ as curvas são mais suaves e levam mais tempo para atingir a taxa de conversão d $\alpha / \mathrm{dt}$ máxima. Em temperaturas maiores $\left(\sim 190-200^{\circ} \mathrm{C}\right)$ as curvas tornam-se mais íngremes e atingem a taxa de reação máxima em um intervalo de tempo mais reduzido. Portanto, temperaturas menores em um ciclo de cura permitem um controle mais apurado da polimerização.

A adição de 2,5 e 5,0\% de PGE na formulação faz com que o sistema fique mais reativo, como pode ser observado na Figura 5(b) e essa reatividade aumenta até a concentração de 5,0\% de PGE, como mostra a Figura 5(c). Observa-se, ainda, na Figura 5c, que as curvas de $d \alpha / \mathrm{dt}$ em função do tempo não são bem definidas e sobrepõem-se em 170 e $180^{\circ} \mathrm{C}$ e depois em 190 e $200^{\circ} \mathrm{C}$.

Para facilitar a comparação dos resultados obtidos na Figura 5 foram construídas curvas de $\mathrm{d} \alpha / \mathrm{dt}$, em função do tempo

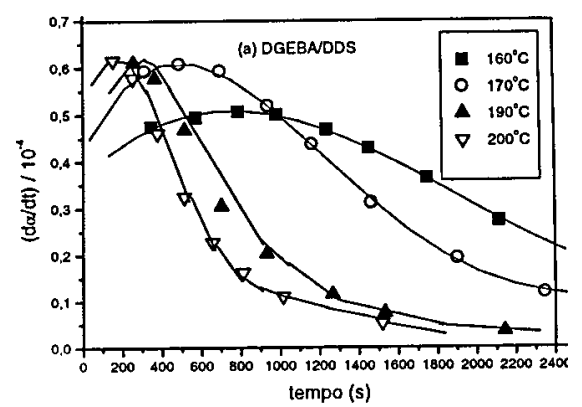

(a)

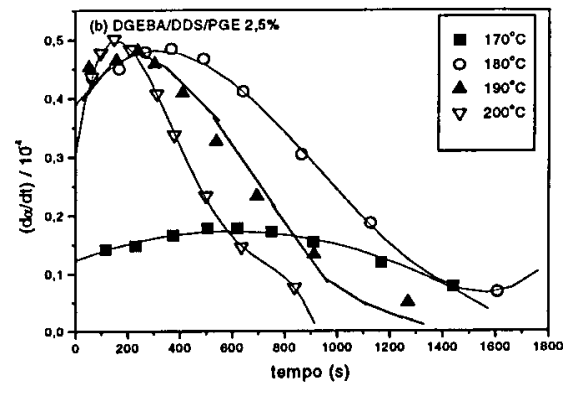

(b)

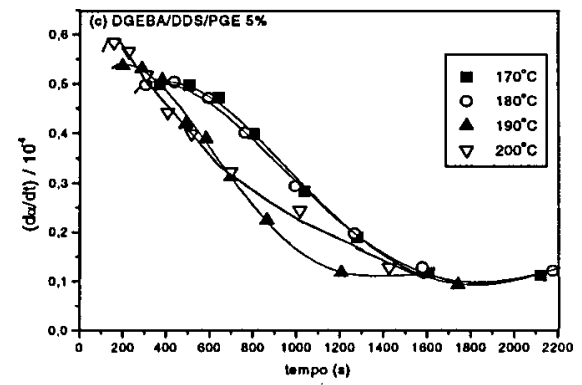

(c)

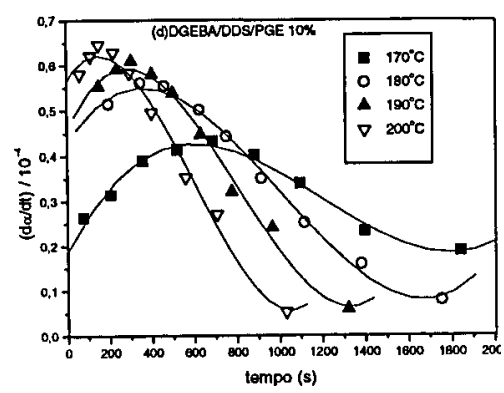

(d)

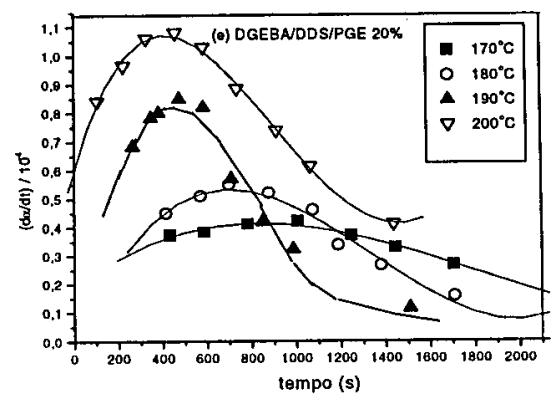

(e)

Figura 5. Taxa de reação isotérmica em função do tempo para quatro temperaturas de isotermas dos sistemas: (a) DGEBA/DDS; (b) PGE 2,5\%, (c) PGE 5\%; (d) PGE 10\% e (e) PGE 20\% 
para uma mesma isoterma $\left(200^{\circ} \mathrm{C}\right)$ contendo todas as formulações (Figura 6). A partir da análise desta Figura é possível verificar que realmente as formulações com 2,5 e $5,0 \%$ de PGE são as mais aceleradas. Isto reforça o que foi mencionado anteriormente na relação dos $\Delta \mathrm{H}_{0}$ dinâmicos (Tabela 3), onde as formulações com PGE 2,5 e 5,0\% são as mais exotérmicas, pois a presença de grupos epóxi provenientes do PGE torna a reação mais acelerada, atingindo a taxa de reação máxima em um curto intervalo de tempo. Esse comportamento indica que as formulações PGE 2,5\% e PGE 5,0\% necessitam de um controle mais rigoroso durante o processamento, principalmente no início da reação de polimerização.

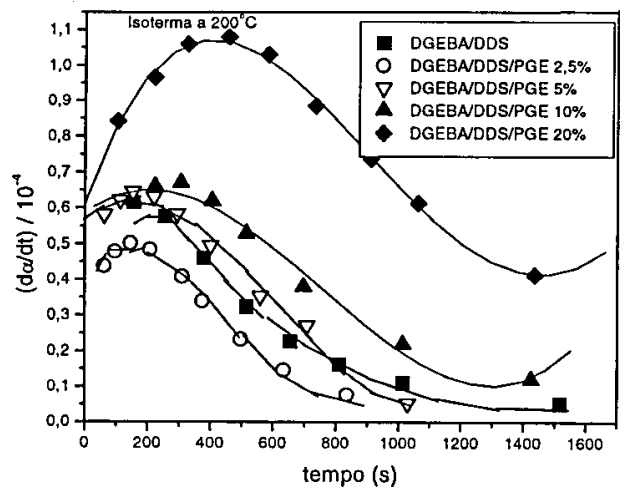

Figura 6. Gráfico da taxa de reação em função do tempo para a temperatura de $200^{\circ} \mathrm{C}$ contendo as cinco formulações.

Já a adição de $10 \%$ de PGE ao sistema faz com que a reação de polimerização fique um pouco mais lenta (Figuras 5 c e 6 ), até mesmo quando comparada com a formulação sem o diluente reativo, DGEBA/DDS (Figuras 5a e 6). E a reação que apresenta o maior tempo de cura é a da formulação PGE20\% (Figuras 5 e e 6). Isso confirma o fato de que um maior número de reações paralelas ocorre, devido à presença de uma maior quantidade de grupos epóxi disponíveis, tornando a reação mais lenta. Dessa maneira, a formulação com $20 \%$ de PGE leva mais tempo para atingir a taxa de reação máxima que os outros sistemas. Esse fato sugere que a presença de PGE em concentrações superiores ou iguais a $20 \%$, para esse sistema, seja uma ferramenta que permite o controle de processamento na manufatura do compósito, produzindo reações mais lentas.

A Figura 7 mostra a relação de Arrhenius das cinco formulações em estudo, ou seja, ln k em função de 1/T (K) utilizada para a determinação da energia de ativação. $O$ coeficiente angular de cada reta representa a energia de ativação para o sistema em questão. As reações químicas em geral têm energia de ativação entre 30 e $100 \mathrm{~kJ} / \mathrm{mol}$. A energia de ativação de sistemas muito viscosos é menor, na faixa de $10-20 \mathrm{~kJ} / \mathrm{mol}^{17}$. Sendo assim, os valores de energia de ativação encontrados 46,$4 ; 44,4 ; 42,4 ; 39,5$ e 37,8 $\mathrm{kJ} / \mathrm{mol}$ estão de acordo com os valores encontrados na literatu$\mathrm{ra}^{18-22}$. A análise do gráfico da Figura 7 mostra que, apesar de se ter avaliado apenas 4 temperaturas diferentes para cada sistema, os valores da energia de ativação são próximos, porém, tendem a diminuir com o aumento da concentração de PGE nos sistemas, devido ao aumento da quantidade de grupos epóxi disponíveis na reação, que diminuem o valor da barreira de energia. Essa observação leva, inicialmente, à conclusão que a reação com maior concentração de PGE seja a mais rápida, uma vez que a sua energia de ativação é a mais baixa.

No entanto, foi determinado experimentalmente que para as formulações com 10 e $20 \%$ de PGE, a conversão é mais baixa (Figura 6). Nesses dois casos em particular, tem-se que a velocidade da reação não é função apenas da energia de ativação, mas também da ordem n, como mostra a equação 4:

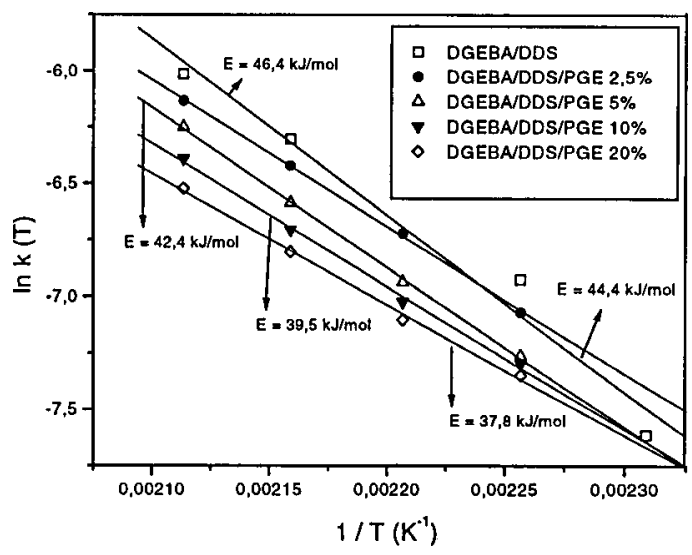

Figura 7. Gráfico de ln $k$ em função de 1/T dos sistemas: DGEBA/ DDS; PGE 2,5\%; PGE 5\%; PGE10\% e PGE $20 \%$.

$\mathrm{v} f\left(\mathrm{E}_{\mathrm{a}}, \mathrm{n}\right)$

Considerando-se que, a energia de ativação das formulações estudadas são próximas, tem-se que:

$\mathrm{v} f(\mathrm{n}), \quad$ se $\mathrm{Ea}=$ cte

Essas observações vêm confirmar o já observado na análise dos valores de $\Delta \mathrm{H}_{0}$, mostrando que esse comportamento devese à ocorrência de um maior número de reações paralelas.

A análise da variação da ordem de reação permite concluir que a mesma aumenta com o aumento da temperatura, como visto nas Tabelas $4,5,6,7$ e 8 .

Para uma determinada temperatura constante tem-se que $\mathrm{n}_{\text {DDS }}>\mathrm{n}_{\text {PGE2 } 2, \%}>\mathrm{n}_{\text {PGE } 5 \%}>\mathrm{n}_{\text {PGE } 10 \%}>\mathrm{n}_{\text {PGE20\% }}$, confirmando os valores de $\Delta \mathrm{H}_{0}$ isotérmicos para uma mesma temperatura onde $\Delta \mathrm{H}_{\text {iso DDS }}>\Delta \mathrm{H}_{\text {iso PGE2,5\% }}>\Delta \mathrm{H}_{\text {iso PGE } 5 \%}>\Delta \mathrm{H}_{\text {iso PGE } 10 \%}>$ $\Delta \mathrm{H}_{\text {isoPGE } 20 \% \text {. }}$

\section{CONCLUSÕES}

O uso da técnica de calorimetria diferencial de varredura confirmou a sua adequação ao estudo da cinética de cura de sistemas de resina epóxi. As formulações estudadas apresentam cinética de polimerização de ordem $n$. Os valores de ordem de reação $\mathrm{n}$ aumentam progressivamente com a temperatura. Observa-se também que, para uma mesma formulação os valores de ordem n diminuem com o aumento da concentração de diluente reativo (PGE) no sistema.

A adição de diluente reativo até a concentração de 5,0\% aumenta a exotermia da reação de polimerização, tornando o sistema mais reativo. Enquanto que, a adição de 10 e $20 \%$ PGE reduz o valor dessa exotermia, fazendo com que a reação de polimerização seja mais lenta. Dados obtidos da taxa de reação $(d \alpha / d t)$ em função do tempo e da conversão $(\alpha)$ confirmam essas observações, mostrando que maiores quantidades de PGE favorecem a presença de um maior número de reações paralelas concorrentes (maior quantidade de grupos no meio reacional), diminuindo a taxa de reação dos sistemas.

As energias de ativação dos cinco sistemas são próximas e estão de acordo com os valores da literatura.

A adição monitorada de diluente reativo mostra-se uma ferramenta adequada para a manufatura dos compósitos estruturais, o que torna o controle de processamento mais fácil, pois produz reações mais lentas.

\section{AGRADECIMENTOS}

Os autores agradecem ao CNPq e à FAPESP pelo suporte financeiro, à empresa Perkin-Elmer pela possibilidade de utili- 
zação do programa de cinética química e à Divisão de Sistemas Bélicos/IAE/CTA por permitir a utilização de seu analisador térmico.

\section{REFERÊNCIAS}

1. Offringa, A. R.; Industrial Reporting, Composites: Part A, 1996, p.329.

2. Newas, G. M.; Polymer matrix composites, in structure and properties of composites. In: Chou, T.W.; Material Science and Technology Series. Ed. VCH Publishers, USA, v.13, 1993.

3. ASM International Comitee.; Enginereed Materials Handbook. V.1: composite, USA, 1987, p.180.

4. Klein, A. J.; Advanced Composites, Cleveland, Ohio, v.3, n.6, 1998, p.36.

5. Otala, M.; Sampe J. 1986, 22, 22.

6. Stover, D.; Advanced Composites, Cleveland, Ohio, v.6, n.5, 1991, p.30.

7. Jang, W.; A review of DSC kinetics methods. Du Pont Instruments.

8. Costa, M. L.; Rezende, M. C. e Pardini, L. C.; Polím: Ciênc. Tecnol. 1999, 2, 37.

9. Berglund, L. A.; Sampe J. 1991, 27, 27.
10. Prime, R. B.; Thermosete, in thermal characterization of polymeric materials. Academic Press, 1981, p.435.

11. Brown, M. E.; Introduction to Thermal Analysis: Techniques and Applications. Ed. Chapman and Hall, USA, 1988, p. 127.

12. Ozawa, T.; J. Therm. Anal. 1970, 2, 301.

13. May, C. A.; Epoxy resins chemistry and technology. Watsonville, C. A.: Marcel Dekker, 1988.

14. The Perkin-Elmer Corporation. Operating Instruction for the DSC 7 Isothermal Kinetics Software Kit. USA, 1994.

15. Byrne, C. A.; Hagnauer, G. L.; Shneider, N. S.; Lenz, R. W.; Polym Compos. 1980, 1, 71.

16. Nam, J.; Seferis, J. C.; J. Appl. Polym. Sci. 1993, 50, 1555.

17. Stutz, H.; Mertes, J.; Neubecker, K.; J. Polym. Sci: Part A: Polym. Chem., 1993, 31, 1879.

18. Miranda, M. I. G.; Tomedi, C.; Bica, I. D.; Samios, D.; Polymer 1997, 38, 1017.

19. Miranda, M. I. G.; Samios, D.; Eur. Polym. J. 1997, 33,325

20. Sykora, V.; Spacek, V.; Dobas, I.; J. Appl. Polym. Sci. 1994, 54, 1463.

21. Souror, S.; Kamal, M. R.; Therm. Acta 1976, 14, 41.

22. Galy, J.; Sabra, A.; Pascault, J. P.; Polym. Eng. Sci. 1986, 26,1514 